Check for updates

Cite this: RSC Adv., 2019, 9, 114

Received 12th September 2018 Accepted 13th November 2018

DOI: $10.1039 / c 8 r a 07470 d$

rsc.li/rsc-advances

\section{Improvement of high-glucose and insulin resistance of chromium malate in 3T3-L1 adipocytes by glucose uptake and insulin sensitivity signaling pathways and its mechanism $\uparrow$}

Weiwei Feng, ${ }^{a}$ Yongchao Liu, ${ }^{a}$ Fan Fei, ${ }^{a}$ Yao Chen, ${ }^{a}$ Yangyang Ding, ${ }^{a}$ Mengjiao Yan, ${ }^{a}$ Yun Feng, ${ }^{\mathrm{b}}$ Ting Zhao, ${ }^{\mathrm{c}}$ Guanghua Mao, ${ }^{\mathrm{a}}$ Liuqing Yang $\mathbb{B}^{* \mathrm{c}}$ and Xiangyang Wu${ }^{\star a}$

Previous study has revealed that chromium malate could improve insulin resistance and the regulation of fasting blood glucose in type 2 diabetic rats. This study was designed to investigate the effect of chromium malate on hypoglycemic and improve insulin resistance activities in 3T3-L1 adipocytes with insulin resistance and investigate the acting mechanism. The result indicated that chromium malate exhibited direct hypoglycemic activity in vitro. Compared with the model group, chromium malate could significantly promote the expression levels of GLUT-4, Akt, Irs-1, PPAR $\gamma$, PI3K and p38-MAPK and their mRNA, increase $p$-AKT/AKT level, AKT and AMPK $\beta 1$ phosphorylation and reduce Irs-1 phosphorylation and $p$-Irs-1/Irs-1 level in 3T3-L1 adipocytes $(p<0.05)$. Chromium malate is more effective in regulating the proteins and mRNA expressions than those of chromium trichloride and chromium picolinate. Compared to the model group, pretreatment with the specific p38-MAPK inhibitor completely inhibited the GLUT-4 and Irs-1 proteins and mRNA expressions induced by the chromium malate. In conclusion, chromium malate had a beneficial influence on improvement of controlling glucose levels and insulin resistance in 3T3-L1 adipocytes with insulin resistance by regulating proteins productions and genes expressions in glucose uptake and insulin sensitivity signaling pathways.

\section{Introduction}

Diabetes is a kind of metabolic disorders and the prevalence of diabetes in adolescents and adults show a fast growth in the world. ${ }^{1,2}$ According to the International Diabetes Federation, from 2013 to 2035 , the number of human with diabetes has increased from 382 million to 592 million. ${ }^{3}$ Type 2 diabetes mellitus (T2DM), which accounts for more than $90 \%$ of diabetes cases, has become a serious public health issue. ${ }^{4}$ Scientific communities have suggested that obesity, air pollution and inheritance could increase the risk of T2DM..$^{5-7}$ The high fasting blood glucose levels and insulin resistance, as the most important factors in the occurrence and development of T2DM, have been commonly found in patients with T2DM.

${ }^{a}$ Institute of Environmental Health and Ecological Security, School of the Environment
and Safety Engineering, Jiangsu University, 301 Xuefu Rd., 212013 Zhenjiang, Jiangsu,
China. E-mail: wuxy@ujs.edu.cn; Fax: +86 $51188791200 ;$ Tel: +86 51188791200
${ }^{b}$ School of Medical Science and Laboratory Medicine, Jiangsu University, 301 Xuefu
Rd., 212013 Zhenjiang, Jiangsu, China
${ }^{c}$ School of Chemistry and Chemical Engineering, Jiangsu University, 301 Xuefu Rd.,
212013 Zhenjiang, Jiangsu, China. E-mail: yangliuqing@ujs.edu.cn; Fax: +86 511
88791800; Tel: +86 51188791800

$\dagger$ Electronic supplementary information (ESI) available. See DOI: 10.1039/c8ra07470d
Chromium (Cr, III) is a beneficial micronutrient. Our previous studies have shown that organic chromium could improve glycometabolism and insulin resistance in T2DM, as well as Brooks et al., Yang et al. and Liu et al. reported..$^{8-11}$ Previous studies suggested that the functionary mechanisms of the anti-hyperglycemic property of organic chromium complexes might be induced by improving glucose uptake and insulin resistance. ${ }^{\mathbf{1 2 - 1 5}}$ However, little research has been reported on systematic studies on the signaling pathways of glucose uptake and insulin sensitivity by organic chromium complex in 3T3-L1 adipocytes with insulin resistance.

In our previous study, chromium malate has been discovered as a safe supplemental or alternative functional food supplement for patients with T2DM. ${ }^{16,17}$ And the antihyperglycemic property and the improving insulin resistance of chromium malate had been found in type 2 diabetic rats, however there were no systematic studies on the mechanism of glucose uptake and insulin sensitivity. Therefore, this study was to discover the possible anti-hyperglycemic and improving insulin resistance mechanisms of chromium malate in 3T3-L1 adipocytes with insulin resistance. The study would be helpful to explore the application value of organic chromium complexes - chromium malate, which could develop a novel functional food or drug. 


\section{Methods}

\section{Materials and chemicals}

The chromium malate was synthesized according to the method described by $\mathrm{Wu}$ et al. ${ }^{18}$ Its chemical formula and molecular weight were $\mathrm{Cr}_{2} \mathrm{C}_{12} \mathrm{H}_{22} \mathrm{O}_{20}$ [or $\left.\mathrm{Cr}_{2}\left(\mathrm{C}_{4} \mathrm{H}_{4} \mathrm{O}_{5}\right)_{3} \cdot 5 \mathrm{H}_{2} \mathrm{O}\right]$ and $590.18 \mathrm{~g}$ $\mathrm{mol}^{-1}$, respectively. The $\mathrm{Cr}$ content is $17.45 \%$. Chromium malate can dissolve in water completely. Standard proteins were purchased from Cell Signaling Technology, Inc (America). Ultrapure water was used throughout the whole experiments.

\section{Cell culture}

3T3-L1 adipocytes were obtained from Institute of Cell Biology, Chinese Academy Sciences (Shanghai, China). Cells were cultured in 95\% air-5\% $\mathrm{CO}_{2}$ atmosphere in DMEM medium supplemented with $10 \% \mathrm{FBS}$ at $37^{\circ} \mathrm{C}$. The differentiated cells were obtained after treating with insulin $\left(10 \mu \mathrm{g} \mathrm{mL}^{-1}\right)$ and IBMX (0.5 mM) for 2 days, insulin $\left(10 \mu \mathrm{g} \mathrm{mL}^{-1}\right)$ for 2 days, $10 \%$ FBSDMEM for 5 days. $^{19,20}$

\section{Models of insulin resistance}

The differentiated cells $\left(2 \times 10^{5}\right.$ cells per $\left.\mathrm{mL}\right)$ were placed in 96 well plates and incubated at $37^{\circ} \mathrm{C}$ for $24 \mathrm{~h}$ in $10 \%$ FBS-DMEM. The cells were incubated with $1 \times 10^{-6} \mathrm{~mol} \mathrm{~L}^{-1}$ insulin for $24 \mathrm{~h}$. $24 \mathrm{~h}$ later, 3T3-L1 adipocytes were washed by DMEM and cultured by $10 \%$ FBS-DMEM, and then the glucose content of supernatant was measured by Glucose oxidase-peroxidase kit. Western blot was used to assay the Irs- 1 and $p$-Irs- 1 productions. The glucose content in 3T3-L1 adipocytes was exhibited significantly higher level than that of normal cells, accompanied with significantly reducing Irs-1 and significantly increasing $p$-Irs-1 was taken as successful induction of insulin resistance in 3T3L1 adipocytes.

\section{Cytotoxicity of chromium malate on 3T3-L1 adipocytes}

The differentiated cells $\left(4 \times 10^{4}\right.$ cells per $\left.\mathrm{mL}\right)$ with insulin resistance were placed in 96-well plates. The various concentrations of chromium malate $(5.0,10.0$ and $20.0 \mu \mathrm{g}$ Cr per $\mathrm{mL}$, low, middle and high dose groups), chromium picolinate (20.0 $\mu \mathrm{g}$ Cr per $\mathrm{mL})$ and chromium trichloride $(20.0 \mu \mathrm{g}$ Cr per $\mathrm{mL})$ were exposed for $24 \mathrm{~h}$. The MTT Kit was used to assay the cytotoxicity of chromium malate. The absorbance was measured in Multimode Reader (SYNERGY H4, BioTek, USA) at $570 \mathrm{~nm}$.

\section{Effect of chromium malate on glucose uptake of 3T3-L1 adipocytes}

The differentiated cells $\left(2 \times 10^{5}\right.$ cells per $\left.\mathrm{mL}\right)$ with insulin resistance were placed in 96-well plates. The various concentrations of chromium malate $(5.0,10.0$ and $20.0 \mu \mathrm{g}$ Cr per $\mathrm{mL}$, low, middle and high dose groups), chromium picolinate and chromium trichloride $(20.0 \mu \mathrm{g}$ Cr per $\mathrm{mL})$ were exposed for $24 \mathrm{~h}$. The 3T3-L1 adipocytes without insulin resistance were exposed to the normal saline and chromium malate $(20.0 \mu \mathrm{g}$ Cr per $\mathrm{mL})$ for $24 \mathrm{~h}$ as control groups. 3T3-L1 adipocytes were washed by
DMEM and cultured by 10\% FBS-DMEM, and then the glucose contents of supernatant were measured by Glucose oxidaseperoxidase kit.

\section{Assay of glucose uptake signaling pathway proteins}

The differentiated cells $\left(2 \times 10^{5}\right.$ cells per $\left.\mathrm{mL}\right)$ with insulin resistance were placed in 6-well plates. The experiment design and the doses of chromium malate, chromium picolinate and chromium trichloride were as same as above described. After incubating for $24 \mathrm{~h}$, the cells were collected. The total protein was extracted and measured. Western blot was used to assay the GLUT-4, $p$-AMPK $\beta 1$ and Akt productions. ${ }^{21,22}$ The blots were visualized and the bands were photographed and analyzed with the Quantity One software.

\section{Assay of insulin sensitivity signaling pathway proteins}

The 3T3-L1 adipocytes cultured, experiment design and the doses of chromium malate, chromium picolinate and chromium trichloride were same as above described. Western blot was used to assay the Irs-1, $p$-Irs-1, PPAR $\gamma$, PI3K and p38-MAPK productions.

\section{Effect of gene expression on glucose uptake and insulin sensitivity signaling pathways}

The 3T3-L1 adipocytes cultured, experiment design and the doses of chromium malate, chromium picolinate and chromium trichloride were as same as above described. After incubating for $24 \mathrm{~h}$, the cells were washed with cold PBS and collected. Real-time PCR (RT-PCR) was used to assay the GLUT4, AMPKa2, Akt2, Irs-1, PPAR $\gamma$, PI3K and p38-MAPK mRNA production. ${ }^{23,24}$ The primers sequence used in this experiment were shown in Table 1.

\section{Effect of receptor protein and gene expression after inhibiting p38MAPK, PI3K and PPAR $\gamma$ expression}

The 3T3-L1 adipocytes cultured and the doses of chromium malate were as same as above described. The 3T3-L1 adipocytes with insulin resistance were exposed to the chromium malate, chromium picolinate, chromium trichloride and GW5074 or LY294002 or GW9662 (3 $\mathrm{MM}$ ) for $24 \mathrm{~h}$. After incubating for $24 \mathrm{~h}$, the cells were collected. The extraction and measure methods of total protein and RNA were as same as above described. Western blot and RT-PCR were used to assay the GLUT-4, Irs-1 and their genes expressions, respectively.

\section{Statistical analysis}

Statistical analyses were carried out using the program SPSS 16.0 (SPSS Inc, Chicago, USA). Averages and standard error were expressed as mean \pm SEM. The one-way analysis of variance (ANOVA) and Tukey test were performed to data analysis and determine the significant differences. A value of $P<0.05$ was considered significantly different statistically. 
Table 1 Sequence of primer used for RT-PCR

\begin{tabular}{lll}
\hline Gene & Forward primer $\left(5^{\prime}\right.$ to $\left.3^{\prime}\right)$ & Reverse primer $\left(3^{\prime}\right.$ to $\left.5^{\prime}\right)$ \\
\hline GAPDH & TGGTGGACCTCATGGCCTAC & CAGCAACTGAGGGCCTCTCT \\
GLUT-4 & GGGCTGTGAGTGAGTGCTTTC & CAGCGAGGCAAGGCTAGA \\
AMPK $\alpha 2$ & GGTGTTATCCTGTATGCCCTTCT & TGTCTTTGATAGTTGCTCGCTTC \\
Akt2 & ATGTAGACTCTCCAGATGAG & TGAGATAATCGAAGTCATTCA \\
Irs-1 & TGTGCCAAGCAACAAGAAAG & ACGGTTCAGAGCAGAGGAA \\
PPAR $\gamma$ & CTTTACCACGGTTGATTTCTC & CAGGCTCTACTTTGATCGCA \\
PI3K & ACTGGAGGAAGACTTGAAG & CGTTTCCCACCATTCGTT \\
p38-MAPK & CGCTGCTGCCGCTGGAAGAT & TTTGGCAATGATGGACT
\end{tabular}

\section{Results and discussion}

\section{Models of insulin resistance}

The glucose contents of insulin induced 3T3-L1 adipocytes were $22.57 \pm 0.64 \mathrm{mmol} \mathrm{L}^{-1}$. The glucose contents in 3T3-L1 adipocytes with insulin resistance were significantly higher than that of normal cells $\left(18.62 \pm 0.59 \mathrm{mmol} \mathrm{L}^{-1}\right)(p<0.05)$. Compared to the normal cells, insulin induced 3T3-L1 adipocytes exhibited significant high levels of glucose $(p<0.05)$. And the Irs-1, AKT, $p$-AKT and $p$-AKT/AKT was significantly reduced, $p$-Irs-1 and $p$-Irs-1/Irs-1, was significantly increased in insulin induced 3T3-L1 adipocytes ( $p<0.05$ ) (ESI Fig. 1†). Consistent with our results, Wang and Yao have also reported that $1 \times$ $10^{-6} \mathrm{~mol} \mathrm{~L}^{-1}$ insulin with 3T3-L1 adipocytes exhibit insulin resistance. ${ }^{\mathbf{1 2}}$ The glucose contents in 3T3-L1 adipocytes were exhibited significant higher level than that of the normal cells, accompanied with Irs-1, AKT, $p$-AKT and $p$-AKT/AKT reduction and $p$-Irs-1 and $p$-Irs-1/Irs-1 increase, which were taken as a successful induction of insulin resistance in 3T3-L1 adipocytes. Therefore, the results confirmed that the 3T3-L1 adipocyte model of insulin resistance is successful.

\section{Cytotoxicity of chromium malate on 3T3-L1 adipocytes}

To assess the cytotoxicity of chromium malate in insulin resistance cells, the 3T3-L1 adipocytes with insulin resistance were incubated with various concentrations of chromium malate (5.0, 10.0 and $20.0 \mu \mathrm{g}$ Cr per $\mathrm{mL}$, low, middle and high dose groups), chromium trichloride and chromium picolinate (20.0 $\mu \mathrm{g}$ Cr per $\mathrm{mL}$ ), respectively. As shown in Fig. 1, compared to the normal control, model and chromium malate control groups, the survival rate of cells did not change significantly in chromium malate (low, middle and high dose groups), chromium trichloride and chromium picolinate groups. High dose of chromium malate did not significantly increase/reduce the survival rate in 3T3-L1 adipocytes with insulin resistance when compared with chromium trichloride and chromium picolinate. The earlier studies reported that $150 \mu \mathrm{M}$ inorganic chromium complex chromium trichloride $(7.8 \mu \mathrm{g}$ Cr per $\mathrm{mL}$ ) could cause supercoiled DNA and linearized DNA molecules in Jurkat cells. ${ }^{25}$ Chromium picolinate is one of the most commonly used organic chromium complexes. However, studies show that $1.5 \mathrm{mM}$ chromium picolinate $(78 \mu \mathrm{g} \mathrm{Cr}$ per $\mathrm{mL})$ could induce $51 \%$ cells undergoing apoptosis and damaged $86 \%$ of the mitochondria. ${ }^{26}$ It can be seen that the cytotoxicity of organic chromium complexes is lesser than those of inorganic chromium complexes. The result suggests that chromium malate had no obvious effect on cytotoxicity in 3T3-L1 adipocytes with insulin resistance at the dose of 0-20.0 $\mu \mathrm{g}$ Cr per $\mathrm{mL}$.

\section{Effect of chromium malate on glucose uptake of 3T3-L1 adipocytes}

The glucose level changes of 3T3-L1 adipocytes with insulin resistance treated with chromium malate were shown in Fig. 2. After exposure to $24 \mathrm{~h}$, the glucose levels of chromium malate in the middle and high dose groups and chromium picolinate group had declined and closed to normal levels (18.41 \pm $\left.0.61 \mathrm{mmol} \mathrm{L}^{-1}\right)$. The glucose levels of the chromium malate groups and model group $\left(22.44 \pm 0.59 \mathrm{mmol} \mathrm{L}^{-1}\right)$ were significantly different $(p<0.05)$. The hypoglycemic activity of chromium malate exhibited a dose dependency. The glucose levels of 3T3-L1 adipocytes with insulin resistance in chromium malate and chromium picolinate groups were decreased significantly when compared with the chromium trichloride group $\left(21.35 \pm 0.43 \mathrm{mmol} \mathrm{L}^{-1}\right)(p<0.05)$. Consistent with our results, Wang and Yao have reported that CrPic could induce glucose uptake in insulin resistance 3T3-L1 adipocytes. ${ }^{12}$ The results confirmed that chromium malate could contribute to glucose uptake and decrease the glucose levels. And the hypoglycemic activity of chromium malate as indicated by glucose levels was superior to that of chromium trichloride. Moreover, exposure of chromium malate had no obvious effect on glucose level of 3T3-L1 adipocytes without insulin resistance.

\section{Assay of glucose uptake signaling pathway proteins}

The possibility of the specific blood glucose accumulation that in turn may negatively affect glucose uptake is represented by high fasting blood glucose (FBG) levels in patients with T2DM. Previous studies have shown that GLUT-4 and AKT could increase the uptake of glucose to enhance the utilization of glucose and reduce the FBG levels. ${ }^{27,28}$ Evidence has suggested that AMPK play a key role in the control of FBG levels in T2DM. ${ }^{29}$ Once activated, AMPK can improve glucose uptake and inhibit glucose production. ${ }^{30,31}$ The abnormal proteins and genes expressions of GLUT-4, AMPK and AKT in the glucose uptake signaling pathway can result in the increasing FBG levels. Therefore, we examined the effects of chromium malate on GLUT-4, $p$-AMPK $\beta 1$, AKT, $p$-AKT and $p$-AKT/AKT (E) levels in insulin resistance 3T3-L1 adipocytes (Fig. 3). Chromium malate could enhance the GLUT- $4, p$-AMPK $\beta 1$, AKT, $p$-AKT and $p$-AKT/ AKT levels in 3T3-L1 adipocytes with insulin resistance. 


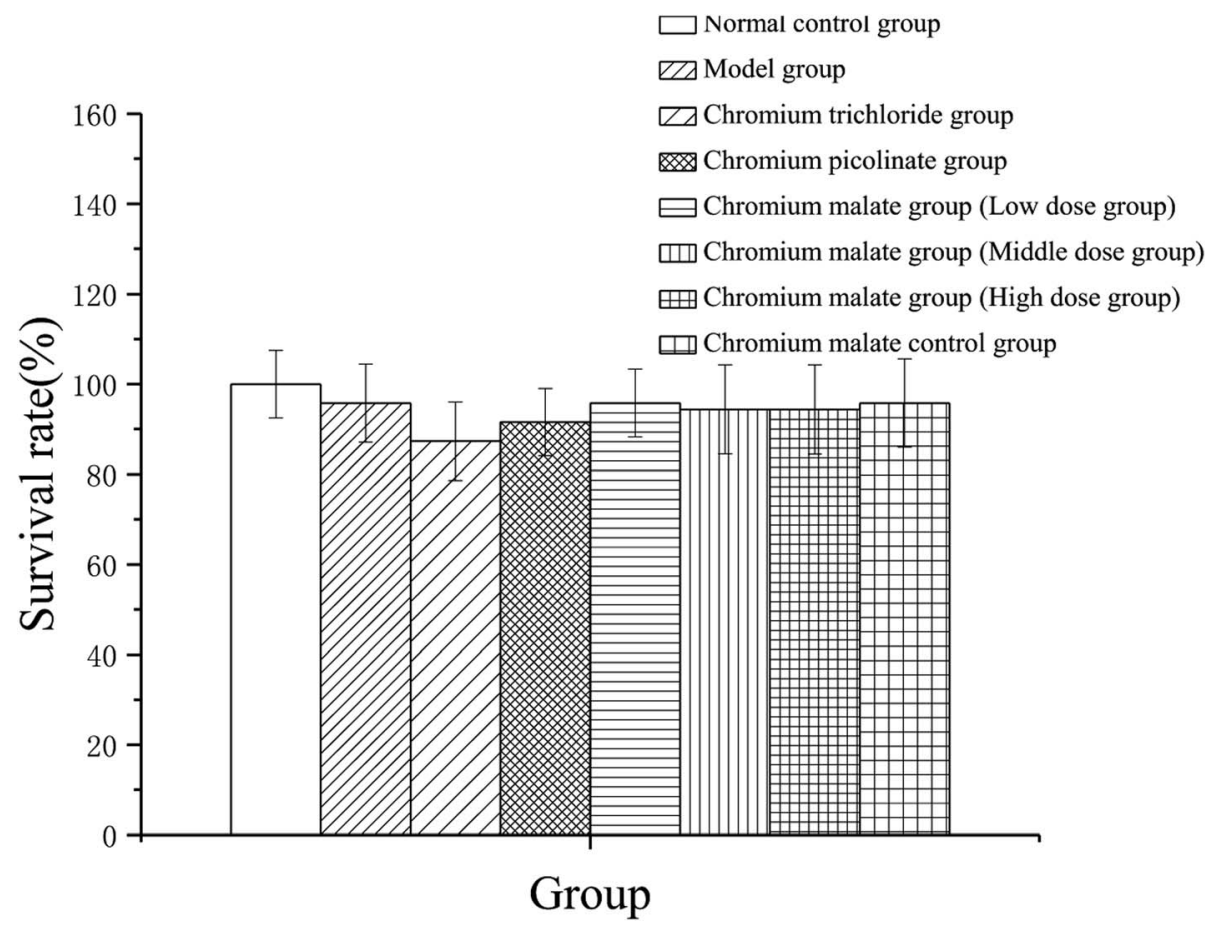

Fig. 1 Effects of chromium malate (5.0,10.0 and $20.0 \mu \mathrm{g} \mathrm{Cr}$ per mL, low, middle and high dose groups) on cytotoxicity in 3T3-L1 cells with insulin resistance. Chromium trichloride $(20.0 \mu \mathrm{g} \mathrm{Cr}$ per $\mathrm{mL})$ and chromium picolinate $(20.0 \mu \mathrm{g} \mathrm{Cr}$ per $\mathrm{mL})$ were used as a positive control. Each value was presented as means $\pm \mathrm{SD}(n=10)$.

Compared with the model group, a significant increase in GLUT-4 and $p$-AKT/AKT levels was observed in chromium malate high dose group and the levels of $p$-AMPK $\beta 1$, AKT and $p$ AKT in chromium malate middle and high dose groups and chromium picolinate group were significantly increased $(p<$ $0.05)$. However, the levels of GLUT- $4, p$-AMPK $\beta 1$, AKT, $p$-AKT and $p$-AKT/AKT in chromium trichloride group had no significant change compared to the model group. Compared with the

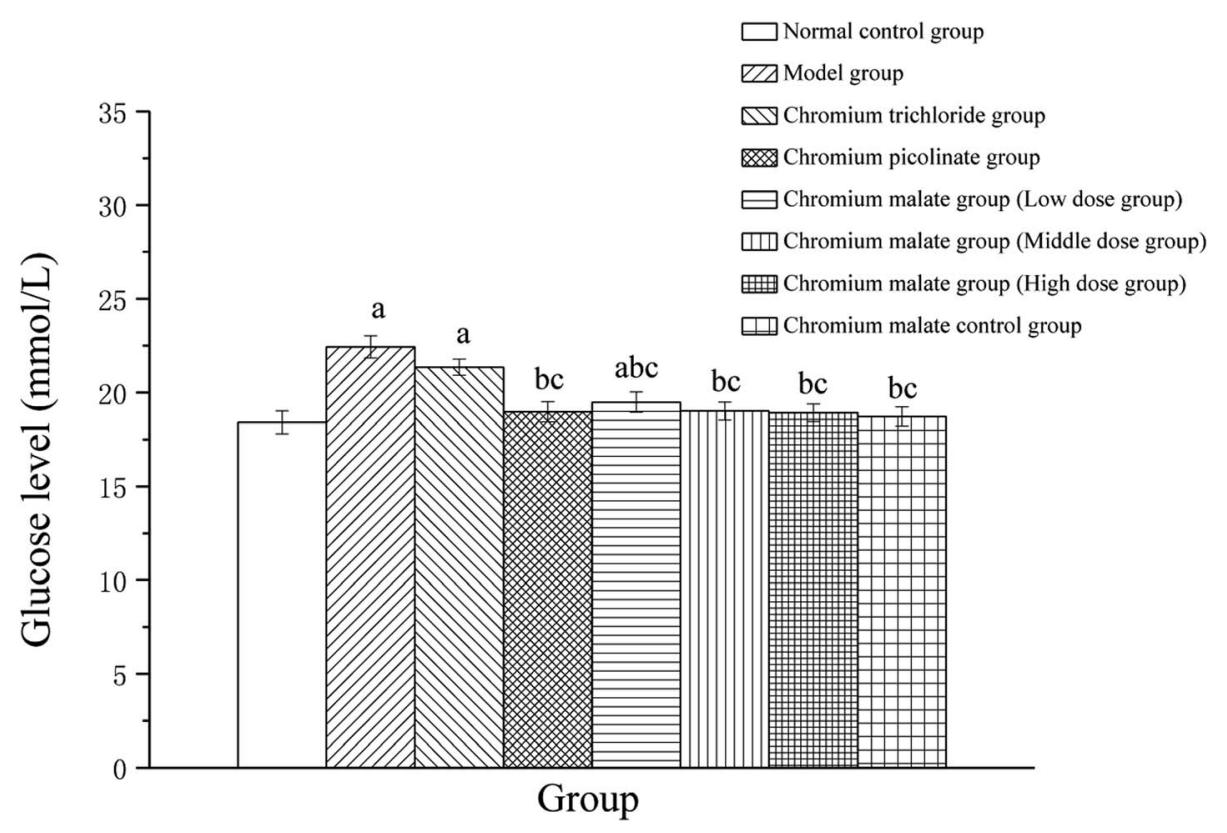

Fig. 2 The glucose level $\left(\mathrm{mmol} \mathrm{L}^{-1}\right)$ changes of 3T3-L1 cells with insulin resistance were treated with chromium malate (5.0, 10.0 and 20.0 $\mu \mathrm{g} \mathrm{Cr}$ per $\mathrm{mL}$, low, middle and high dose groups). Chromium trichloride (20.0 $\mu \mathrm{g} \mathrm{Cr}$ per $\mathrm{mL})$ and chromium picolinate $(20.0 \mu \mathrm{g} \mathrm{Cr}$ per $\mathrm{mL}) \mathrm{were}$ used as a positive control. Each value was presented as means \pm SD $(n=10)$. ${ }^{a}$ Significantly different from normal group $(p<0.05)$; ${ }^{b}$ significantly different from model group $(p<0.05)$; ${ }^{c}$ significantly different from chromium trichloride group $(p<0.05)$; ${ }^{d}$ significantly different from chromium picolinate group $(p<0.05)$. 

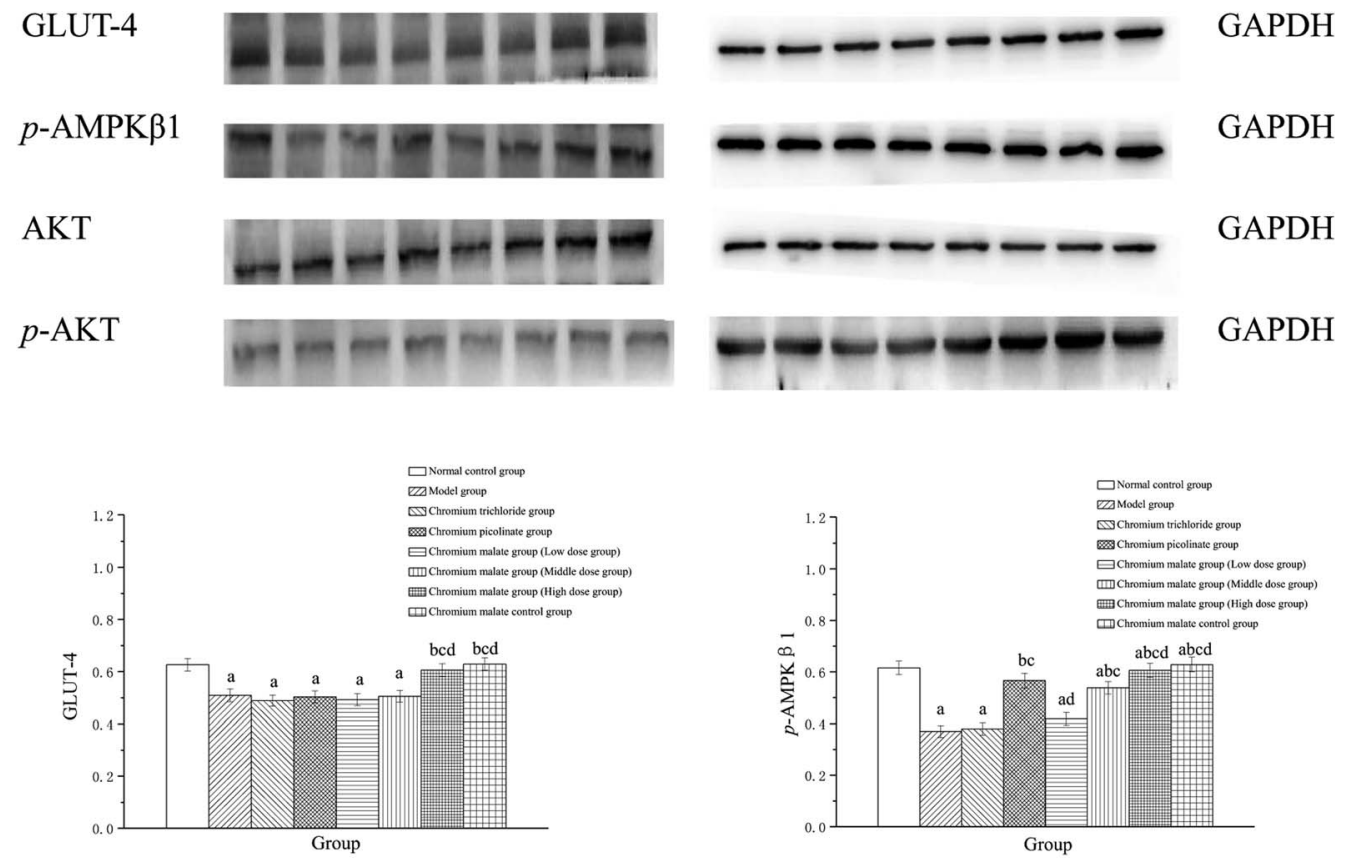

A

B
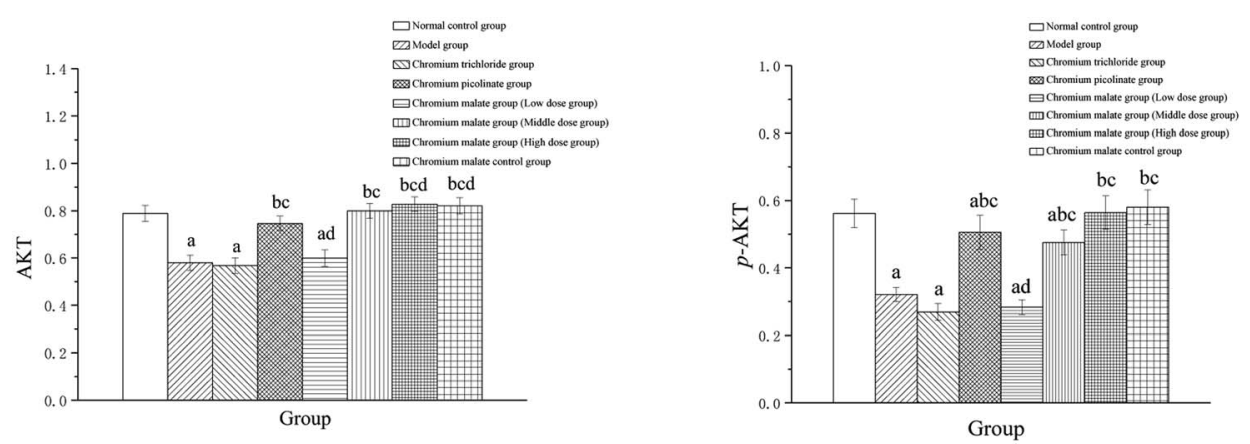

C

D

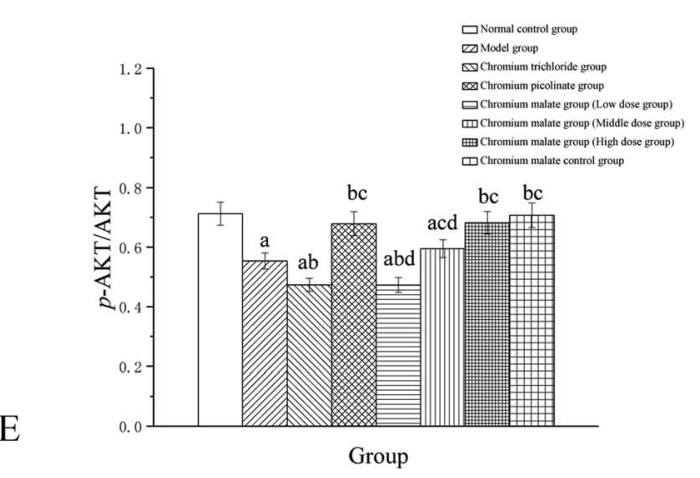

Fig. 3 Effect of chromium malate (5.0, 10.0 and $20.0 \mu \mathrm{g}$ Cr per mL, low, middle and high dose groups) on GLUT-4 (A), p-AMPK 31 (B), AKT (C), $p$ AKT (D) expression and $p$-AKT/AKT (E) level in 3T3-L1 cells with insulin resistance. Chromium trichloride $(20.0 \mu \mathrm{g} \mathrm{Cr}$ per mL) and chromium picolinate $(20.0 \mu \mathrm{g} \mathrm{Cr}$ per $\mathrm{mL})$ were used as a positive control. Each value was presented as means $\pm \mathrm{SD}(n=10)$. ${ }^{\mathrm{S}} \mathrm{Significantly}$ different from normal group ( $p<0.05)$; ' ${ }^{b}$ ignificantly different from model group $(p<0.05)$; ${ }^{c}$ significantly different from chromium trichloride group $(p<0.05)$; dsignificantly different from chromium picolinate group $(p<0.05)$ 
model group, the $p$-AMPK $\beta 1$, Akt, $p$-AKT and $p$-AKT/AKT levels of 3T3-L1 adipocytes in chromium picolinate group with insulin resistance increased significantly $(p<0.05)$. The levels of GLUT$4, p$-AMPK $\beta 1$, Akt, $p$-AKT and $p$-AKT/AKT in chromium malate high dose group were significantly increased compared to the chromium trichloride group $(p<0.05)$. The chromium malate control group could not significantly enhance the levels of GLUT- $4, p$-AMPK $\beta 1$, AKT, $p$-AKT and $p$-AKT/AKT when compared with the normal control group. Therefore, chromium malate had no obvious effect on the GLUT-4, $p$-AMPK $\beta 1$, Akt, $p$ AKT and $p$-AKT/AKT levels of 3T3-L1 adipocytes without insulin resistance. Jovanovic et al. reported that Co-Factor III Cr Yeast could provoke a significant increase of the GLUT-4 and AMPK expressions and AKT phosphorylation in Holstein calves. ${ }^{32}$ Peng et al. reported that chromium phenylalanine could sensitize signaling pathway in cells with insulin resistance via the activation of Akt and AMPK. ${ }^{33}$ The literatures shown that the organic chromium complexes could improve glucose uptake in model organism. These results suggested that chromium malate could exhibit its glucose uptake activity by regulating glucose uptake signaling pathways proteins productions through up-regulation of GLUT-4 and Akt, promoting AMPK $\beta 1$ and AKT phosphorylation and $p$-AKT/AKT ratio. And chromium malate is more effective in regulating GLUT-4, $p$-AMPK $\beta 1$, Akt and $p$-AKT than those of chromium trichloride and chromium picolinate.

\section{Assay of insulin sensitivity signaling pathway proteins}

The insulin sensitivity signaling pathway can modulate insulin resistance in T2DM, and its impairment is one of the main molecular mechanisms of T2DM. ${ }^{34}$ It is involved in Irs-1, PPAR $\gamma$, PI3K and p38-MAPK genes and proteins expressions. Previous studies have suggested that IRS-1 and PI3K are key molecules in insulin sensitivity signaling pathway. ${ }^{35,36}$ The lower levels of IRS-1 and PI3K in insulin signal transduction pathway with defects were found in T2DM. ${ }^{37}$ Peroxisome proliferatoractivated receptor gamma (PPAR gamma) can enhance insulin sensitivity in muscle and adipose tissues, and some of exogenous potent PPAR $\gamma$ ligands are used as medicines to T2DM. ${ }^{38}$ p38-MAPK can regulate glucose metabolism. However, the activation of the p38-MAPK can result in phosphorylation of the insulin receptor and IRS proteins, leading to impaired insulin signaling. ${ }^{39}$ Therefore, we examined the effects of chromium malate on Irs-1, $p$-Irs-1, $p$-Irs-1/Irs-1, PPAR $\gamma$, PI3K and p38MAPK levels in insulin resistance 3T3-L1 adipocytes. Effects of chromium malate on Irs-1, $p$-Irs-1, $p$-Irs-1/Irs-1, PPAR $\gamma$, PI3K and p38-MAPK levels in 3T3-L1 adipocytes with insulin resistance were presented in Fig. 4. Chromium malate could enhance the levels of Irs-1, PPAR $\gamma$, PI3K and p38-MAPK, and reduce $p$-Irs-1 and $p$-Irs-1/Irs-1 levels in 3T3-L1 adipocytes with insulin resistance. Compared with the model group, chromium malate showed significant increase in the Irs-1 and PPAR $\gamma$ levels, significant reduction in the $p$-Irs-1 and $p$-Irs-1/Irs-1 levels in low, middle and high dose groups and significant increase in the PI3K and p38-MAPK levels in middle and high dose groups $(p<0.05)$. Chromium malate increased the productions of Irs-1,
PPAR $\gamma$, PI3K and p38-MAPK, and decreased the Irs-1 phosphorylation and $p$-Irs-1/Irs-1 in dose-independent manners. The group exposed to chromium picolinate showed significant increase in Irs-1, PPAR $\gamma$ and PI3K levels, and significant decrease in $p$-Irs- 1 and $p$-Irs-1/Irs-1 levels except for p38-MAPK production, when compared with the model group $(p<0.05)$. The results of the chromium trichloride group showed that no significant changes were observed in Irs-1, $p$-Irs-1, PPAR $\gamma$, PI3K and p38-MAPK productions. Comparing the levels of the Irs-1, $p$-Irs-1, $p$-Irs-1/Irs-1, PPAR $\gamma$, PI3K and p38-MAPK with the chromium picolinate and chromium trichloride groups, the results showed that a significant change occurred in chromium malate high dose group $(p<0.05)$. The chromium malate could not significantly change the levels of Irs-1, $p$-Irs-1, $p$-Irs-1/Irs-1, PPAR $\gamma$, PI3K and p38-MAPK in chromium malate control group when compared with the normal control group. Therefore, chromium malate had no obvious effect on the Irs-1, $p$-Irs1, $p$-Irs-1/Irs-1, PPAR $\gamma$, PI3K and p38-MAPK levels of 3T3-L1 adipocytes without insulin resistance. These results suggested that chromium malate could improve insulin resistance by regulating insulin sensitivity signaling pathway proteins production through up-regulation of Irs-1, $p$-Irs-1, PPAR $\gamma$, PI3K and p38-MAPK, and down-regulation of Irs- 1 phosphorylation and $p$-Irs-1/Irs- 1 . And chromium malate is more effective in regulating Irs-1, $p$-Irs-1, $p$-Irs-1/Irs-1, PPAR $\gamma$, PI3K and p38MAPK levels than those of chromium trichloride and chromium picolinate.

\section{Effect of gene expression on glucose uptake signaling pathway}

Effects of chromium malate on the GLUT-4, AMPK $\alpha 2$ and AKT2 mRNA expression levels of 3T3-L1 adipocytes with insulin resistance were showed in Fig. 5. Chromium malate could enhance the mRNA expressions of GLUT-4, AMPK $\alpha 2$ and Akt 2 in 3T3-L1 adipocytes with insulin resistance. Compared to the model group, the mRNA expression levels of GLUT-4 and AKT2 in chromium malate low, middle and high dose groups and the mRNA expression levels of AMPKa2 in chromium malate middle and high dose groups were significantly increased $(p<$ 0.05). Chromium malate increased the mRNA expressions of GLUT-4, AMPK $\alpha 2$ and Akt 2 in a dose dependency manner. The mRNA expression levels of GLUT-4, AMPKa2 and Akt2 of 3T3-L1 adipocytes with insulin resistance in chromium picolinate group were increased significantly when compared with the model group $(p<0.05)$. However, the GLUT-4, AMPK $\alpha 2$ and Akt2 mRNA expression in chromium trichloride group had no significant change. Compared with the chromium picolinate and chromium trichloride groups, the mRNA expressions of GLUT-4, AMPK $\alpha 2$ and Akt2 in chromium malate high dose group were increased significantly $(p<0.05)$. The mRNA expressions of GLUT-4, AMPK $\alpha 2$ and Akt2 had no significant change between chromium malate control group and normal control group. Therefore, chromium malate had no obvious effect on the GLUT-4, AMPK $\alpha 2$ and Akt2 mRNA expressions of 3T3-L1 adipocytes without insulin resistance. Consistent with our results, Hao et al. have reported that oligomannuronate chromium complex could enhance the mRNA expression of 

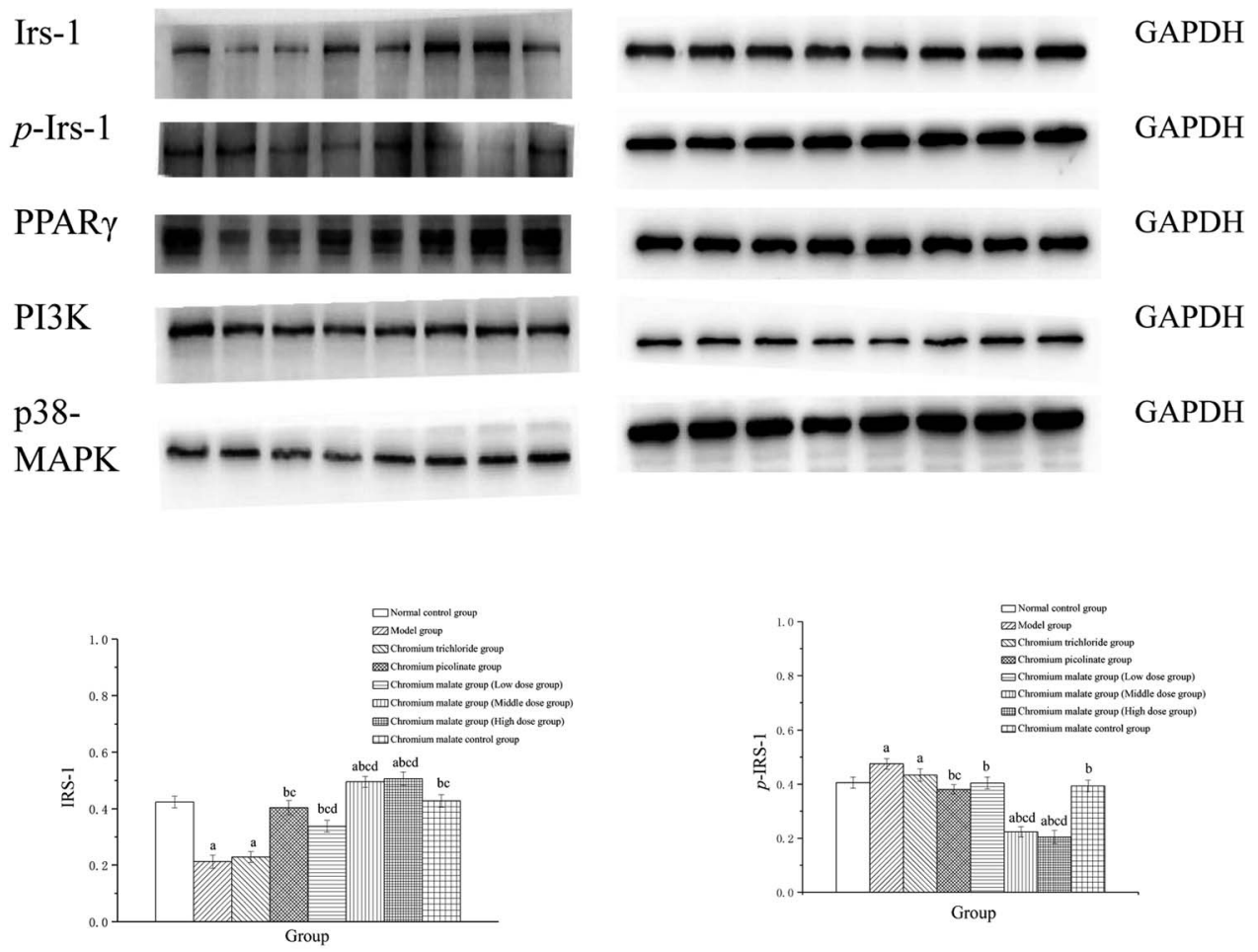

A
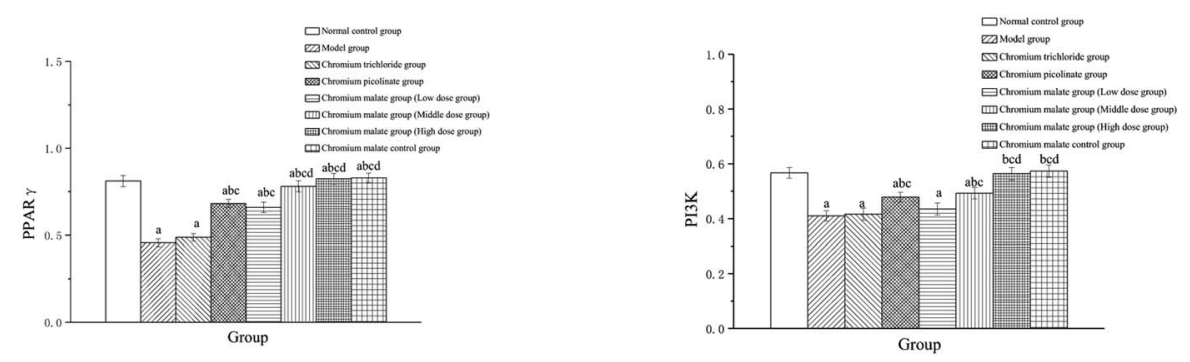

C

D
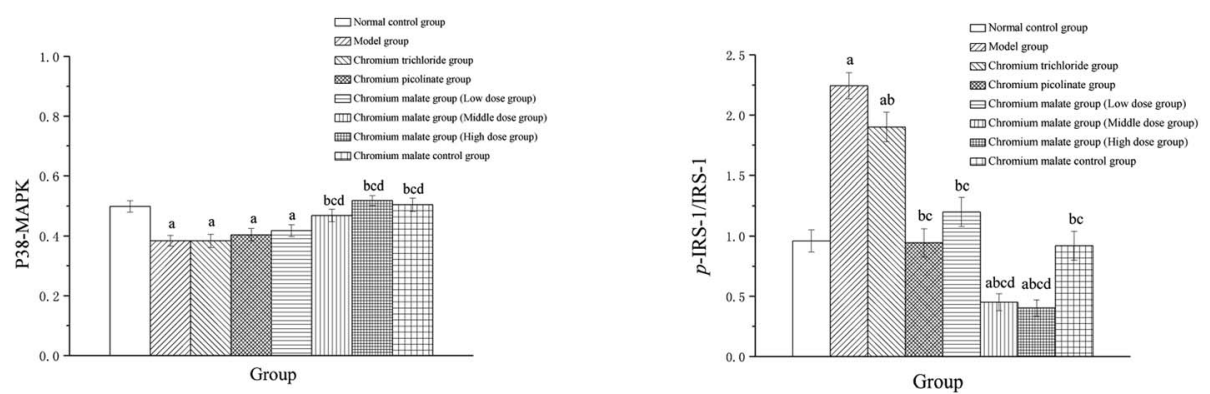

E

F

Fig. 4 Effect of chromium malate (5.0, 10.0 and $20.0 \mu \mathrm{g}$ Cr per mL, low, middle and high dose groups) on Irs-1 (A), p-Irs-1 (B), PPAR (C), PI3K (D) and p38-MAPK (E) expression and $p$-Irs-1/Irs-1 (F) level in 3T3-L1 cells with insulin resistance. Chromium trichloride (20.0 $\mu \mathrm{g} \mathrm{Cr}$ per $\mathrm{mL}$ ) and chromium picolinate $\left(20.0 \mu \mathrm{g} \mathrm{Cr}\right.$ per $\mathrm{mL}$ ) were used as a positive control. Each value was presented as means $\pm \mathrm{SD}(n=10)$. ${ }^{\mathrm{a} S i g n i f i c a n t l y}$ different from normal group ( $p<0.05)$; ${ }^{b}$ significantly different from model group $(p<0.05)$; ${ }^{c}$ significantly different from chromium trichloride group $(p<0.05)$; ${ }^{d}$ significantly different from chromium picolinate group $(p<0.05)$. 


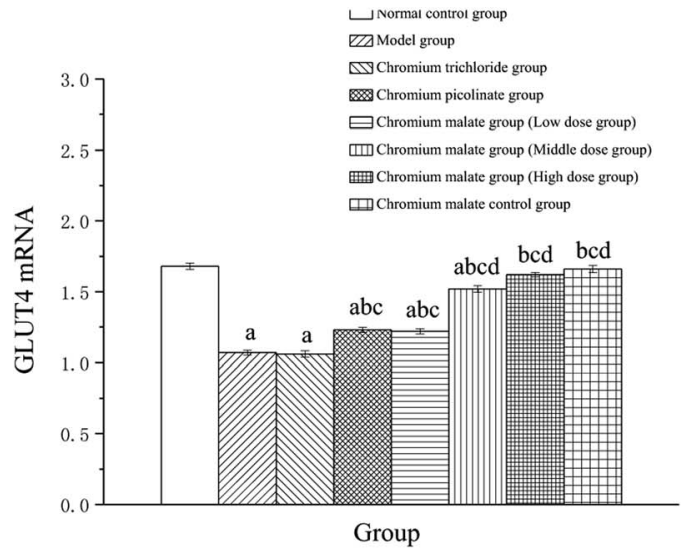

A

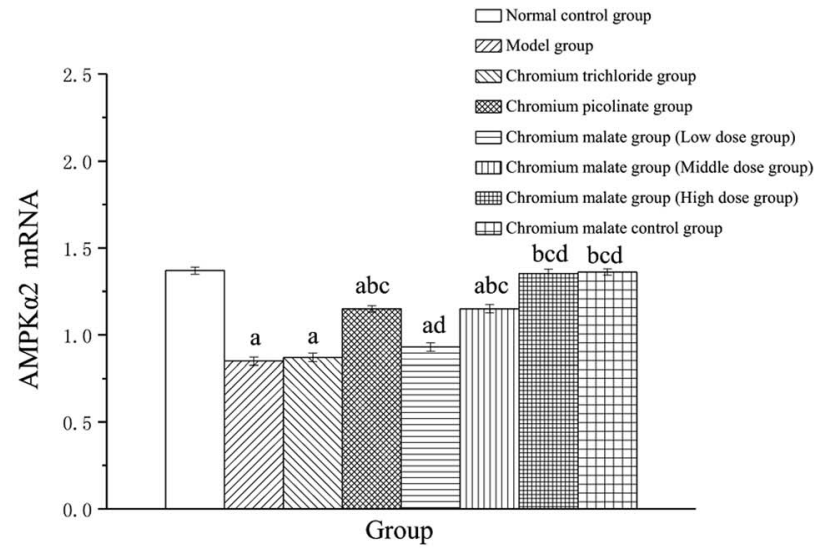

$\mathrm{B}$

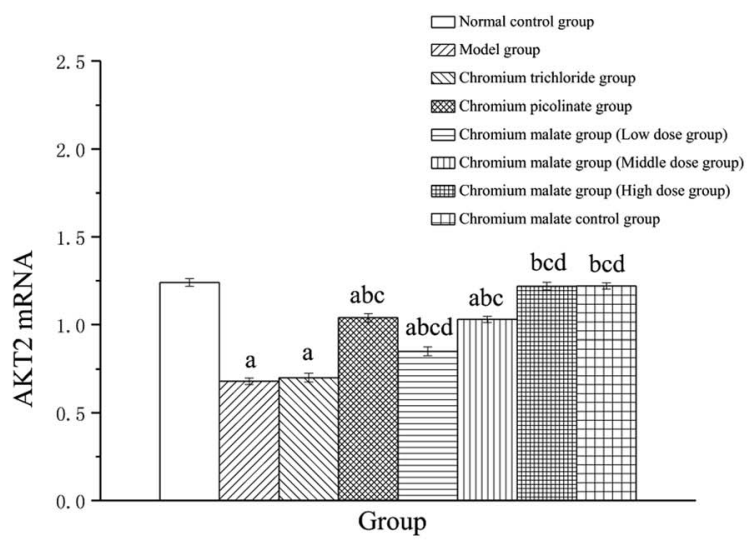

C

Fig. 5 Effect of chromium malate (5.0, 10.0 and $20.0 \mu \mathrm{g} \mathrm{Cr}$ per mL, low, middle and high dose groups) on the GLUT-4 (A), AMPK 22 (B) and Akt2 (C) mRNA expression level of 3T3-L1 cells with insulin resistance. Chromium trichloride $(20.0 \mu \mathrm{g} \mathrm{Cr}$ per $\mathrm{mL}$ ) and chromium picolinate (20.0 $\mu \mathrm{g} \mathrm{Cr}$

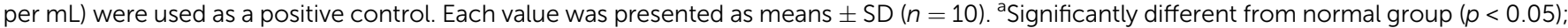
${ }^{b}$ significantly different from model group $(p<0.05)$; ${ }^{c}$ significantly different from chromium trichloride group $(p<0.05)$; ${ }^{d}$ significantly different from chromium picolinate group $(p<0.05)$.

GLUT-4 in $\mathrm{C}_{2} \mathrm{C}_{12}$ skeletal muscle cells with insulin resistance. ${ }^{13}$ These results revealed that chromium malate could exhibit its glucose uptake activity by regulating gene expressions of receptor proteins on glucose uptake signaling pathway through up-regulation of GLUT-4, AMPK $\alpha 2$ and Akt2 mRNA. And chromium malate is more effective in regulating GLUT-4, AMPK $\alpha 2$ and Akt2 mRNA expressions than those of chromium trichloride and chromium picolinate.

\section{Effect of gene expression on insulin sensitivity signaling pathway}

Effects of chromium malate on the Irs-1, PPAR $\gamma$, PI3K and p38MAPK mRNA expression levels of 3T3-L1 adipocytes with insulin resistance were presented in Fig. 6. Chromium malate could enhance the mRNA expressions of Irs-1, PPAR $\gamma$, PI3K and
p38-MAPK in 3T3-L1 adipocytes with insulin resistance. Compared with the model group, chromium malate showed significant increase in the mRNA expressions levels of Irs-1, PPAR $\gamma$ and p38-MAPK in low, middle and high dose groups and significant increase in the mRNA expression levels of PI3K in middle and high dose groups $(p<0.05)$. Chromium malate increased the mRNA expressions of Irs-1, PPAR $\gamma$, PI3K and p38MAPK in dose-dependent manners. Chromium picolinate group showed significant increase in the mRNA expressions of Irs-1, PPAR $\gamma$, PI3K and p38-MAPK when compared with the model group $(p<0.05)$. However, chromium trichloride group showed no significant change in the mRNA expressions of Irs-1, PPAR $\gamma$, PI3K and p38-MAPK. Comparing the mRNA expression levels of the Irs-1, PPAR $\gamma$, PI3K and p38-MAPK in the chromium picolinate and chromium trichloride groups, the results showed 


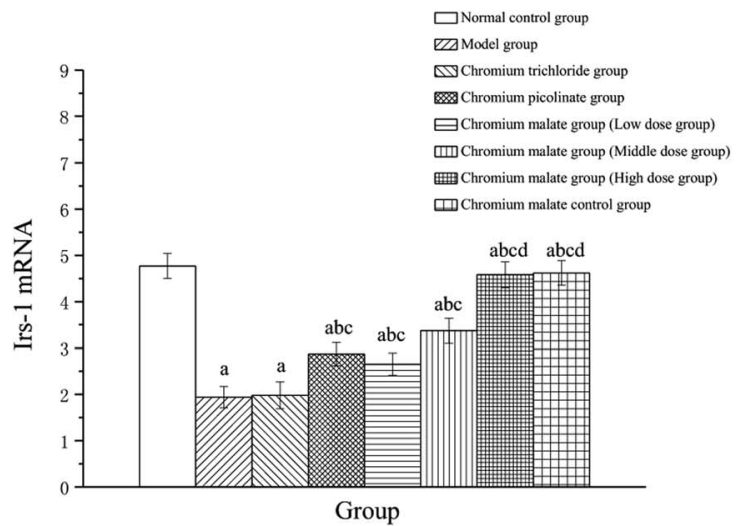

A

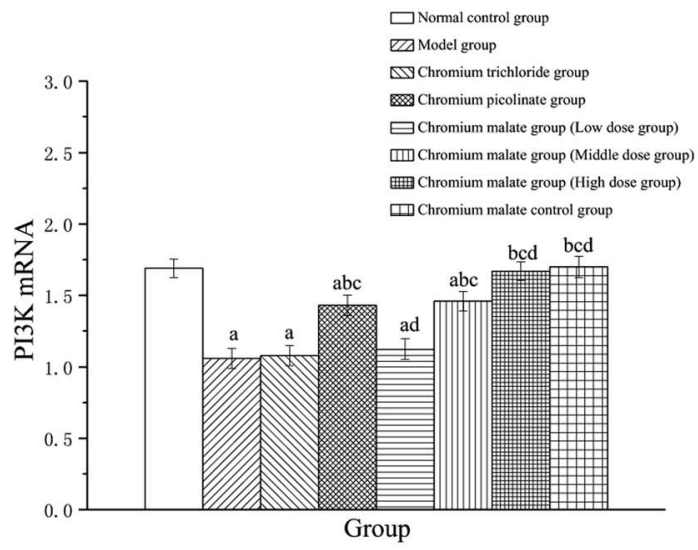

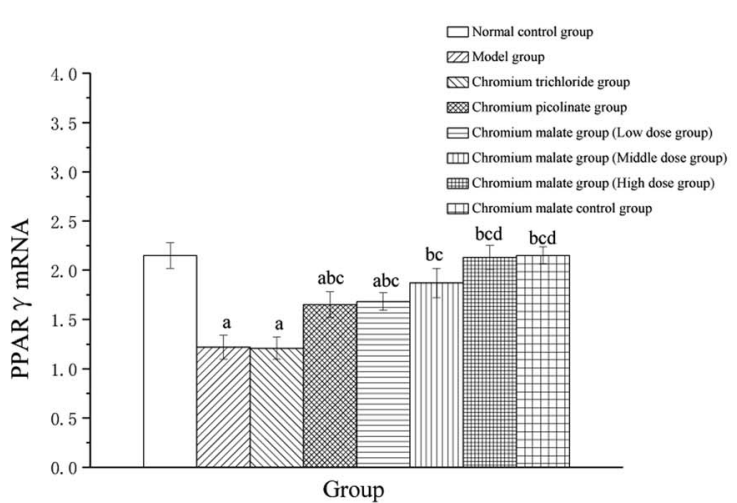

B

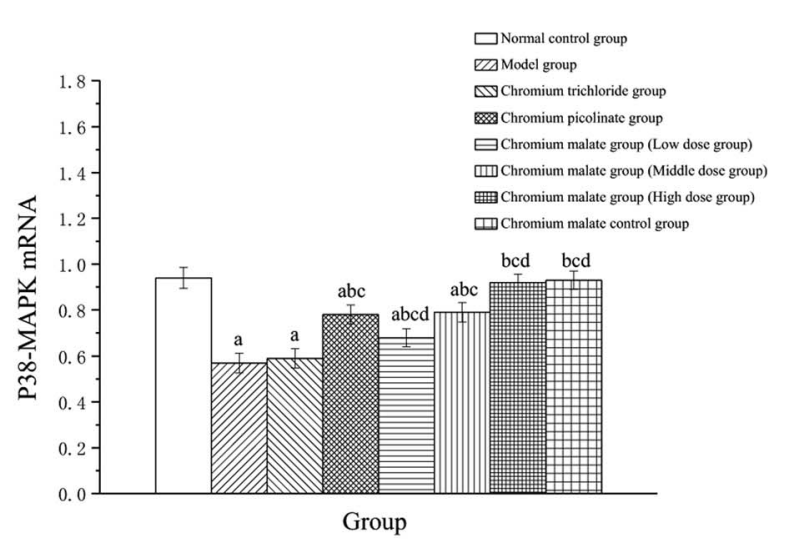

C

D

Fig. 6 Effect of chromium malate (5.0, 10.0 and $20.0 \mu \mathrm{g} \mathrm{Cr}$ per mL, low, middle and high dose groups) on the Irs-1 (A), PPAR (B), PI3K (C) and p38-MAPK (D) mRNA expression levels of 3T3-L1 cells with insulin resistance. Chromium trichloride $(20.0 \mu \mathrm{g} \mathrm{Cr}$ per $\mathrm{mL})$ and chromium picolinate

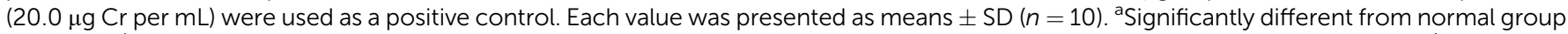
$(p<0.05)$; ${ }^{b}$ significantly different from model group $(p<0.05)$; ${ }^{c}$ significantly different from chromium trichloride group $(p<0.05)$; ${ }^{d}$ significantly different from chromium picolinate group $(p<0.05)$.

that a significant increase was observed in chromium malate high dose group $(p<0.05)$. The mRNA expression levels of Irs-1, PPAR $\gamma$, PI3K and p38-MAPK had no significant change between chromium malate control group and normal control group. Therefore, chromium malate had no obvious effect on the Irs-1, PPAR $\gamma$, PI3K and p38-MAPK mRNA expressions of 3T3-L1 adipocytes without insulin resistance. Liu et al. reported that chromium yeast could improve glucose metabolism in insulinresistant 3T3-L1 adipocytes via increase Irs-1 mRNA. ${ }^{11} \mathrm{~A}$ similar expression trend was observed for PPAR $\gamma$ mRNA in 3T3-L1 adipocytes treatment with $\mathrm{Cr}(\mathrm{III})$-citrate. ${ }^{\mathbf{4 0}}$ These results suggested that chromium malate could improve insulin resistance by regulating gene expressions of receptor proteins on insulin sensitivity signaling pathway through up-regulation of Irs-1, PPAR $\gamma$, PI3K and p38-MAPK mRNA. And chromium malate is more effective in regulating Irs-1, PPAR $\gamma$, PI3K and p38-MAPK mRNA expressions than those of chromium trichloride and chromium picolinate.

\section{Models of inhibiting p38 MAPK, PI3K and PPAR $\gamma$ expression}

Compared to the 3T3-L1 adipocytes with insulin resistance, GW5074 or LY294002 or GW9662 induced significantly low levels of p38 MAPK, PI3K and PPAR $\gamma$ in 3T3-L1 adipocytes ( $p<$ 0.05) (ESI Fig. $2 \dagger$ ). The p38 MAPK, PI3K and PPAR $\gamma$ levels in 3T3-L1 adipocytes with insulin resistance exhibited significant inhibitions than that of the insulin resistance cells without corresponding inhibitors, which were taken as successful inhibited p38 MAPK, PI3K and PPAR $\gamma$ expression. Therefore, the results confirmed that the models of inhibiting p38 MAPK, PI3K and PPAR $\gamma$ expression are successful.

\section{Effect of receptor protein and gene expression after inhibiting p38MAPK expression}

Effect of receptor protein production after inhibiting p38MAPK expression. Effects of chromium malate on the GLUT4 and Irs-1 expression levels of 3T3-L1 adipocytes with insulin 
resistance when p38MAPK expression was inhibited were shown in Fig. $7 \mathrm{~A}(1)$ and $\mathrm{B}(1)$. After inhibiting p38-MAPK expression, the levels of GLUT-4 and Irs-1 in chromium malate control group were lower than that of normal control group. The results confirmed that the p38-MAPK was inhibited successfully in 3T3-L1 adipocytes. Compared with the normal control group, various doses of chromium malate groups, chromium trichloride group and chromium picolinate group showed significant decrease in the levels of GLUT-4 and Irs-1 ( $p$ $<0.05)$. Compared to the model group, there was no significant effect on increase of GLUT-4 and Irs-1 in chromium malate low dose group. However, chromium malate showed significant increase in the production levels of GLUT- 4 and Irs- 1 in middle and high dose groups $(p<0.05)$. The productions of GLUT- 4 and Irs-1 in chromium malate high dose group were significantly increased compared to the chromium trichloride and chromium picolinate groups $(p<0.05)$.

Effect of gene expression after inhibiting p38MAPK expression. Effects of chromium malate on the GLUT-4 (A) and Irs-1 (B) mRNA expression levels of 3T3-L1 adipocytes with insulin

\section{GLUT-4}

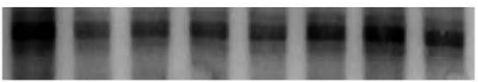

Irs-1

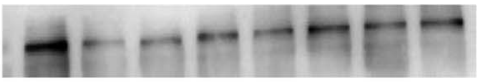

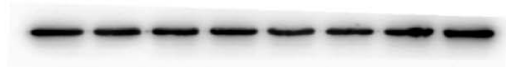

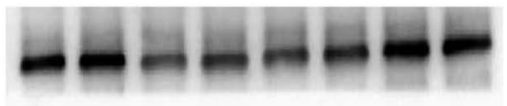

\section{GAPDH}

\section{GAPDH}

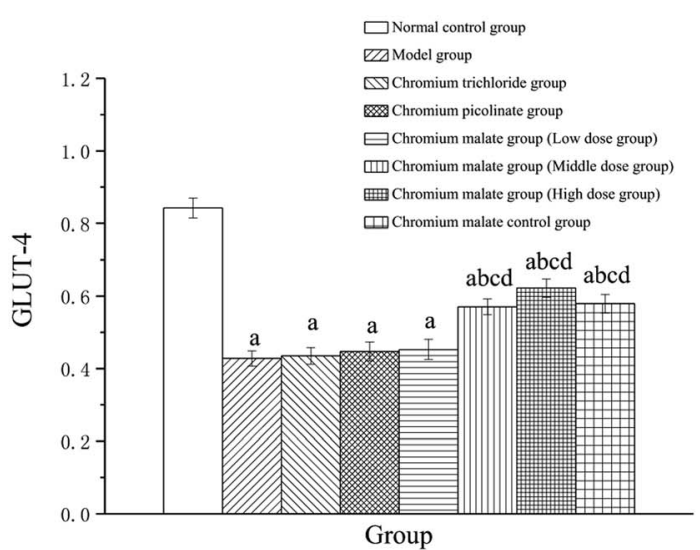

$\mathrm{A}(1)$

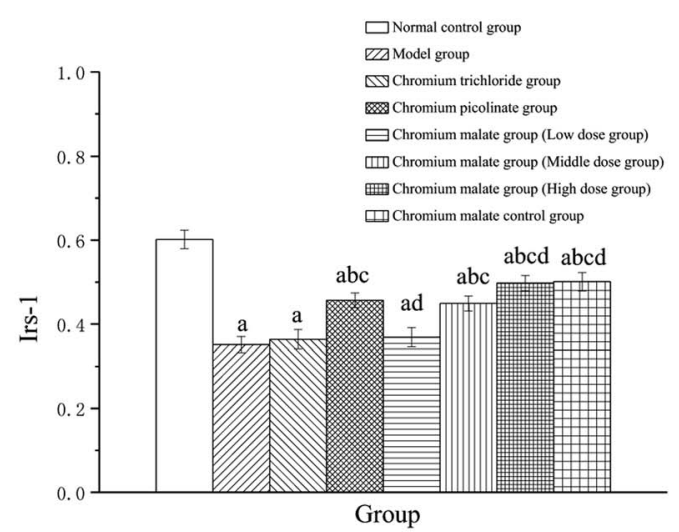

$\mathrm{B}(1)$

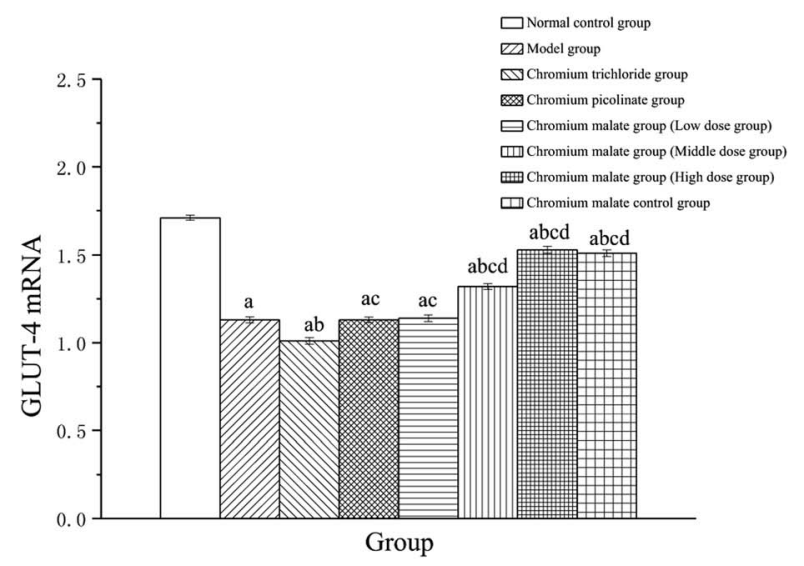

$\mathrm{A}(2)$

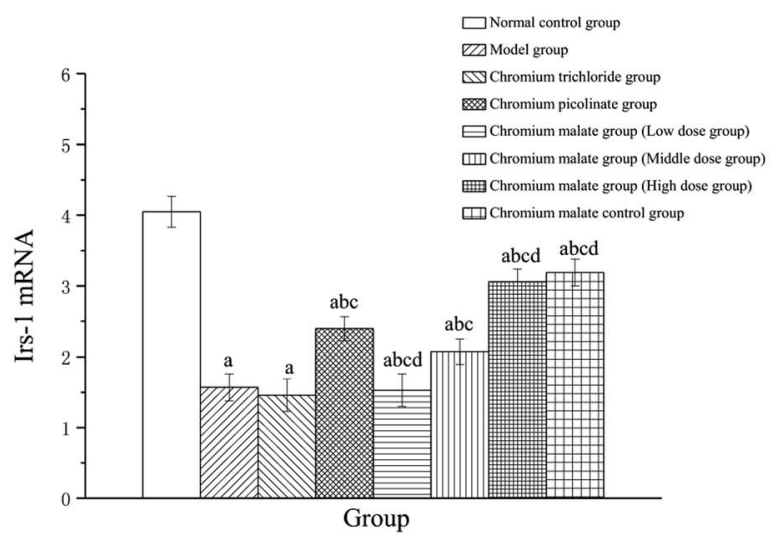

$\mathrm{B}(2)$

Fig. 7 Effect of chromium malate (5.0, 10.0 and $20.0 \mu \mathrm{g} \mathrm{Cr}$ per mL, low, middle and high dose groups) on the GLUT-4 (A1) and Irs-1 (B1) expression levels and GLUT-4 (A2) and Irs-1 (B2) mRNA expression levels of 3T3-L1 cells with insulin resistance when block p38MAPK expression (GW5074, $3 \mu \mathrm{M})$. Chromium trichloride $(20.0 \mu \mathrm{g} \mathrm{Cr}$ per $\mathrm{mL})$ and chromium picolinate $(20.0 \mu \mathrm{g} \mathrm{Cr}$ per mL) were used as a positive control. Each

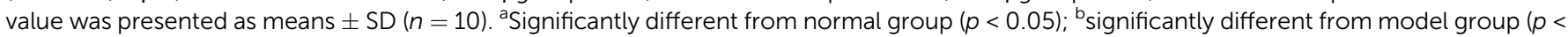
0.05); " significantly different from chromium trichloride group $(p<0.05)$; ${ }^{d}$ significantly different from chromium picolinate group $(p<0.05)$. 


\section{GLUT-4}

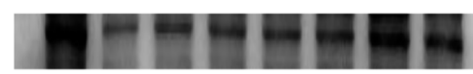

Irs-1

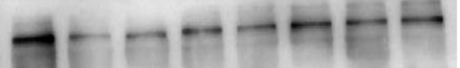

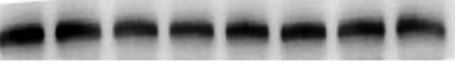

\section{GAPDH}

\section{GAPDH}

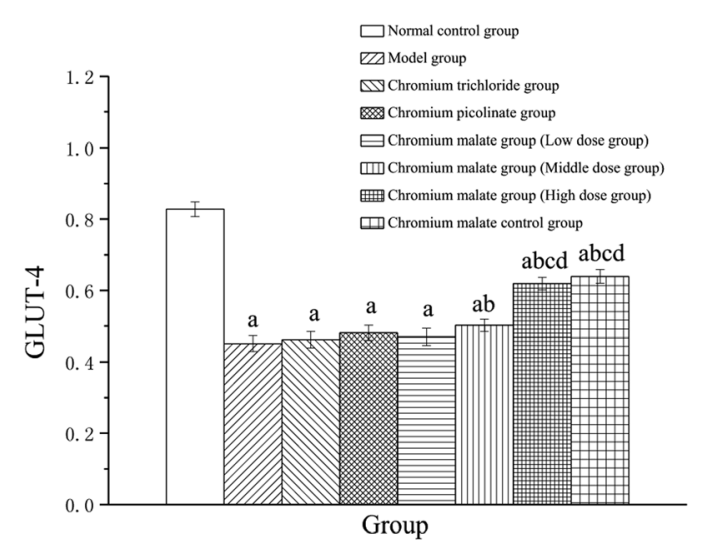

A(1)

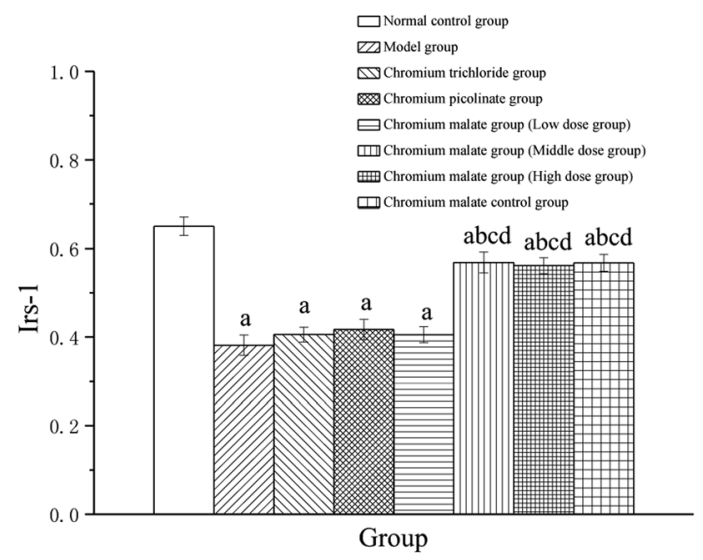

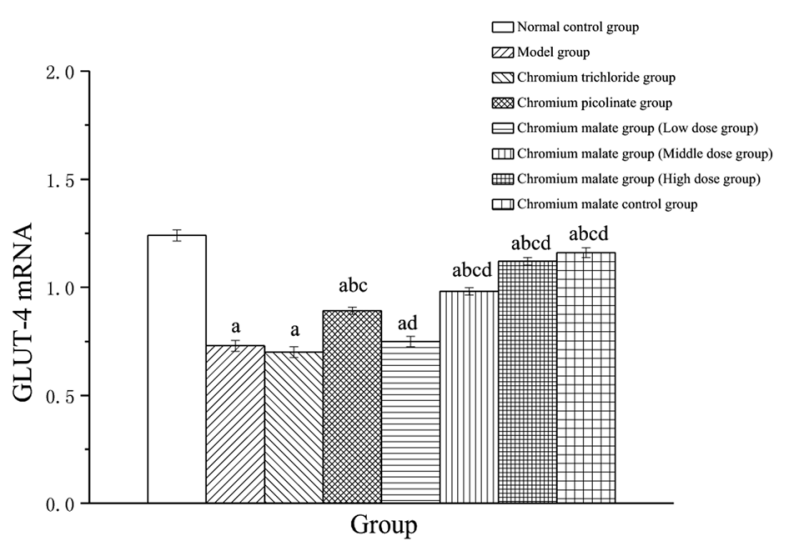

$\mathrm{A}(2)$

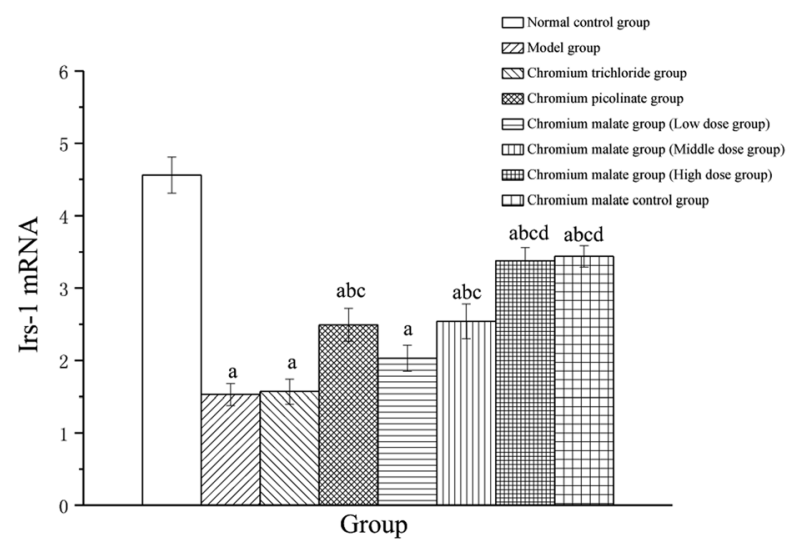

$\mathrm{B}(2)$

Fig. 8 Effect of chromium malate (5.0, 10.0 and $20.0 \mu \mathrm{g} \mathrm{Cr}$ per mL, low, middle and high dose groups) on the GLUT-4 (A1) and Irs-1 (B1) expression levels and GLUT-4 (A2) and Irs-1 (B2) mRNA expression levels of 3T3-L1 cells with insulin resistance when block PI3K expression $(\mathrm{LY} 294002,3 \mu \mathrm{M})$. Chromium trichloride $(20.0 \mu \mathrm{g} \mathrm{Cr}$ per $\mathrm{mL})$ and chromium picolinate $(20.0 \mu \mathrm{g} \mathrm{Cr}$ per $\mathrm{mL})$ were used as a positive control. Each

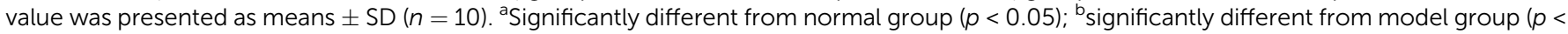
0.05); ' significantly different from chromium trichloride group $(p<0.05)$; ${ }^{d}$ significantly different from chromium picolinate group $(p<0.05)$.

resistance when p38-MAPK expression was inhibited were shown in Fig. $7 \mathrm{~A}(2)$ and $\mathrm{B}(2)$. After inhibiting p38-MAPK expression, the GLUT-4 and Irs-1 mRNA expressions of chromium malate control group in 3T3-L1 adipocytes with insulin resistance were lower than that of normal control group. The results confirmed that the p38-MAPK was inhibited successfully in 3T3-L1 adipocytes. Various doses of chromium malate, chromium trichloride and chromium picolinate significantly reduced the mRNA expressions of GLUT-4 and Irs- 1 when compared to normal control group $(p<0.05)$. Compared to the model group, there was no significant change in GLUT-4 and Irs-1 mRNA expressions in the chromium malate low dose group. However, chromium malate showed significant increase in the mRNA expression levels of GLUT-4 and Irs- 1 in middle 
GLUT-4

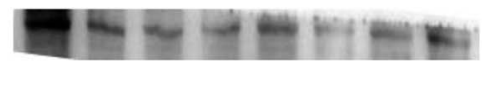

Irs-1
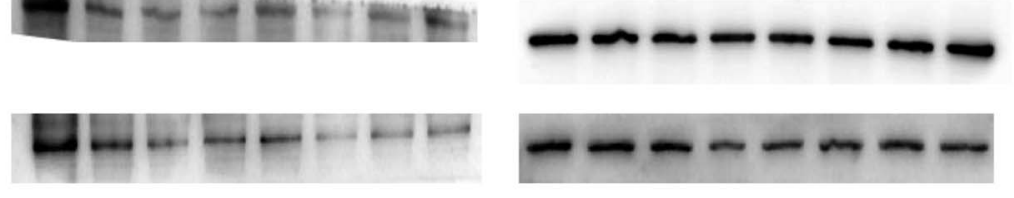

\section{GAPDH}

GAPDH

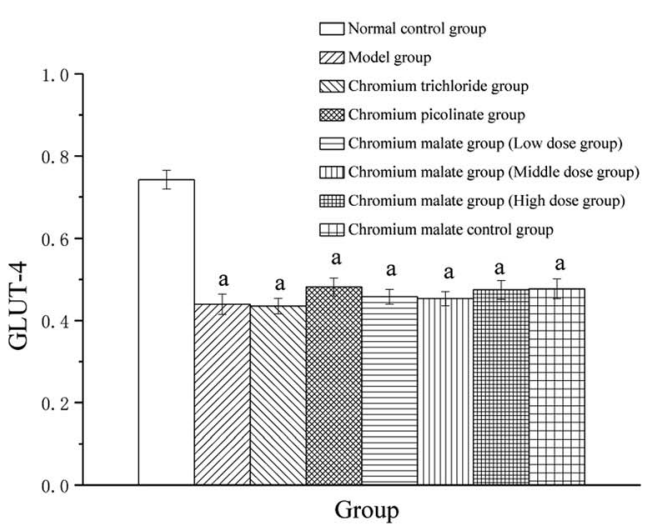

A(1)

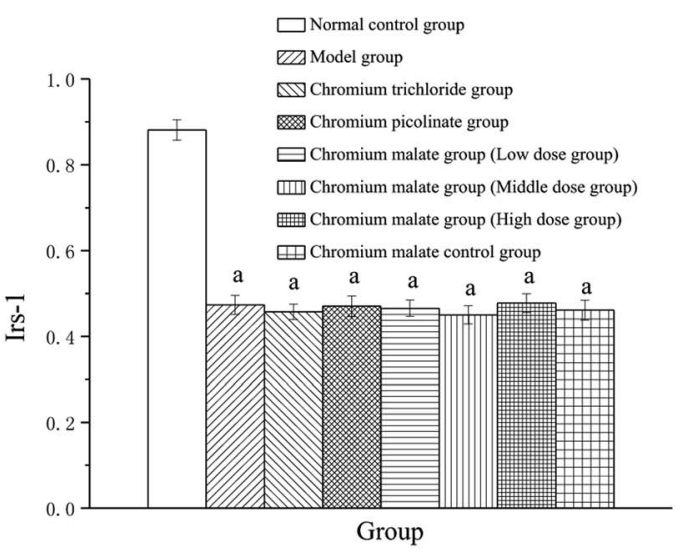

$\mathrm{B}(1)$

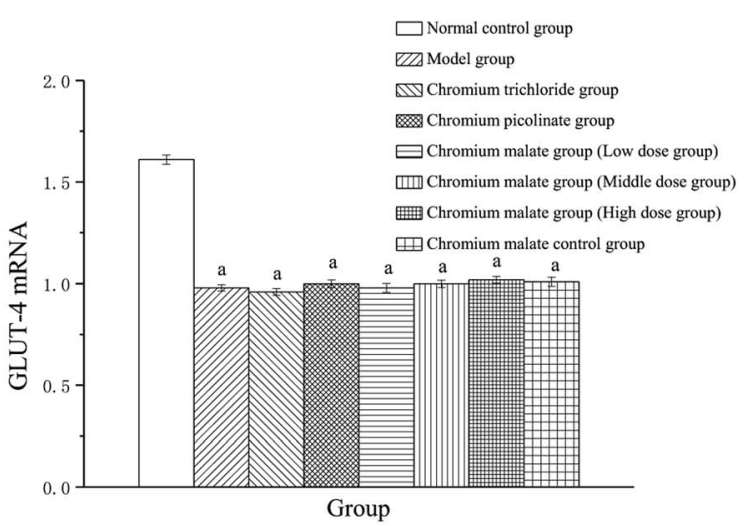

$\mathrm{A}(2)$

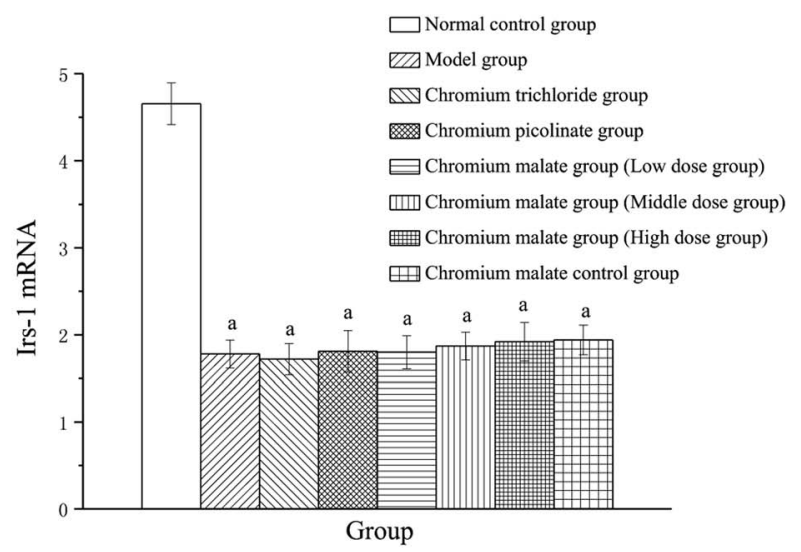

$\mathrm{B}(2)$

Fig. 9 Effect of chromium malate (5.0, 10.0 and $20.0 \mu \mathrm{g} \mathrm{Cr}$ per mL, low, middle and high dose groups) on the GLUT-4 (A1) and Irs-1 (B1) expression levels and GLUT-4 (A2) and Irs-1 (B2) mRNA expression levels of 3T3-L1 cells with insulin resistance when block PPAR $\gamma$ expression (GW9662, $3 \mu \mathrm{M})$. Chromium trichloride $(20.0 \mu \mathrm{g} \mathrm{Cr}$ per $\mathrm{mL}$ ) and chromium picolinate $(20.0 \mu \mathrm{g} \mathrm{Cr}$ per $\mathrm{mL})$ were used as a positive control. Each value was presented as means \pm SD $(n=10)$. ${ }^{a}$ Significantly different from normal group $(p<0.05)$.

and high dose groups $(p<0.05)$. Moreover, high dose of chromium malate increased the mRNA expression levels of GLUT-4 and Irs-1 more than chromium trichloride and chromium picolinate.

These results suggested that chromium malate had hypoglycemic activity and could improve insulin resistance in 3T3-L1 adipocytes with insulin resistance by regulating p38-MAPKinduced proteins productions and genes expressions. And the hypoglycemic activity and the improvement of insulin resistance of chromium malate were not only through regulating p38-MAPK-induced proteins production and genes expression, but also through regulating other key protein-induced proteins productions and genes expressions.

\section{Effect of receptor protein and gene expression after inhibiting PI3K expression}

Effect of receptor protein production after inhibiting PI3K expression. Effects of chromium malate on the GLUT-4 and Irs1 expression levels of 3T3-L1 adipocytes with insulin resistance when PI3K expression was inhibited were presented in Fig. 8A(1) and B(1). After inhibiting PI3K expression, significant 
reductions of GLUT-4 and Irs-1 expressions were observed in chromium malate control group when compared with the normal control group $(p<0.05)$. The results confirmed that the PI3K was inhibited successfully in 3T3-L1 adipocytes. Compared with the normal control group, significant reductions of GLUT-4 and Irs-1 productions were found in various doses of chromium malate, chromium trichloride and chromium picolinate groups $(p<0.05)$. Low dose of chromium malate showed no significant change in the levels of GLUT-4 and Irs-1 when compared to the model group. However, middle and high doses of chromium malate showed significant increase in the production levels of GLUT- 4 and Irs- $1(p<0.05)$. The productions of GLUT- 4 and Irs- 1 in chromium malate high dose group were significantly increased when compared with the chromium trichloride and chromium picolinate groups $(p<$ 0.05).

Effect of gene expression after inhibiting PI3K expression. Effects of chromium malate on the GLUT-4 (A) and Irs-1 (B) mRNA expression levels of 3T3-L1 adipocytes with insulin resistance after inhibiting PI3K expression were showed in Fig. $8 \mathrm{~A}(2)$ and $\mathrm{B}(2)$. Compared to the normal control group, the mRNA expression levels of GLUT-4 and Irs-1 in chromium malate control group were significantly reduced after inhibiting PI3K expression $(p<0.05)$. The results confirmed that the PI3K was inhibited successfully in 3T3-L1 adipocytes. Compared with the normal control group, significant reductions of GLUT-4 and Irs-1 mRNA expression in various doses of chromium malate, chromium trichloride and chromium picolinate groups were found $(p<0.05)$. However, middle and high doses of chromium malate showed significant increase in the mRNA expression levels of GLUT-4 and Irs-1 $(p<0.05)$. Moreover, high dose of chromium malate could significantly increase the mRNA expression levels of GLUT- 4 and Irs- 1 than those of chromium trichloride and chromium picolinate $(p<0.05)$.

These results suggested that chromium malate could improve high-glucose levels and insulin resistance in 3T3-L1 adipocytes with insulin resistance by regulating PI3K-induced proteins productions and genes expressions. And the hypoglycemic activity and improving insulin resistance of chromium malate were not only through regulating PI3K-induced proteins productions and genes expressions, but also through regulating other key protein-induced proteins productions and genes expressions.

\section{Effect of receptor protein and gene expression after inhibiting PPAR $\gamma$ expression}

Effect of receptor protein production after inhibiting PPAR $\gamma$ expression. Effects of chromium malate on the GLUT-4 (A) and Irs-1 (B) expression levels of 3T3-L1 adipocytes with insulin resistance after inhibiting PPAR $\gamma$ expression were showed in Fig. 9A(1) and $\mathrm{B}(1)$. Compared to the normal control group, the production levels of GLUT-4 and Irs-1 in chromium malate control group were significantly reduced after inhibiting PPAR $\gamma$ expression $(p<0.05)$. The results confirmed that the PPAR $\gamma$ was inhibited successfully in 3T3-L1 adipocytes. The productions of GLUT-4 and Irs-1 in various doses of chromium malate, chromium trichloride and chromium picolinate groups were significantly reduced when compared with the normal control group $(p<0.05)$. However, there was no significant change compared to the model group. Various doses of chromium malate groups showed no significant change in the productions of GLUT- 4 and Irs- 1 when compared with the chromium trichloride and chromium picolinate groups.

Effect of gene expression after inhibiting PPAR $\gamma$ expression. Effects of chromium malate on the GLUT-4 (A) and Irs-1 (B) mRNA expression levels of 3T3-L1 adipocytes with insulin resistance after inhibiting PPAR $\gamma$ expression were showed in Fig. $9 \mathrm{~A}(2)$ and $\mathrm{B}(2)$. Compared with the normal control group, the mRNA expression levels of GLUT-4 and Irs-1 in chromium malate control group were significantly reduced after inhibiting PPAR $\gamma$ expression $(p<0.05)$. The results confirmed that the PPAR $\gamma$ was inhibited successfully in 3T3-L1 adipocytes. Compared with the model group, the mRNA expressions levels of GLUT-4 and Irs-1 in various doses of chromium malate, chromium trichloride and chromium picolinate groups were not significantly increased. However, there was a significant reduction when compared with normal control group $(p<0.05)$. Various dose of chromium malate groups showed no significant changes in the mRNA expression levels of GLUT-4 and Irs-1 compared to chromium trichloride and chromium picolinate groups.

These results suggested that chromium malate could improve high-glucose levels and insulin resistance in 3T3-L1 adipocytes with insulin resistance by regulating PPAR $\gamma$ induced proteins productions and genes expressions.

\section{Conclusion}

Chromium malate exhibited direct hypoglycemic activity in vitro and greater benefits in treating 3T3-L1 adipocytes with insulin resistance induced high-glucose levels than that of chromium trichloride. Chromium malate could induce 3T3-L1 adipocytes with insulin resistance to express GLUT-4, Akt, Irs-1, PPAR $\gamma$, PI3K and p38-MAPK proteins and mRNA, increase AMPK $\beta 1$ phosphorylation and $p$-AKT/AKT level and reduce Irs-1 phosphorylation and $p$-Irs-1/Irs-1 level. The results of pretreatment with the specific p38MAPK/PI3K/PPAR $\gamma$ inhibitor showed that the chromium malate could improve high-glucose levels and insulin resistance in 3T3-L1 adipocytes with insulin resistance by regulating proteins productions and genes expressions in glucose uptake and insulin sensitivity signaling pathways.

\section{Conflicts of interest}

No.

\section{Acknowledgements}

This work was supported financially by Specialized Research Fund for the Natural Science Foundation of China (31271850) and Research Foundation for Advanced Talents of Jiangsu University (15JDG146). 


\section{References}

1 F. Akhlaghi, K. L. Matson, A. H. Mohammadpour, M. Kelly and A. Karimani, Clin. Pharmacokinet., 2017, 56, 561-571.

2 World Health Organization (WHO), http://apps.who.int/iris/ bitstream/10665/204871/1/9789241565257_eng.pdf, 2016, accessed November 2016.

3 L. Guariguata, D. R. Whiting, I. Hambleton, J. Beagley, U. Linnenkamp and J. E. Shaw, Diabetes Res. Clin. Pract., 2014, 13, 137-149.

4 Y. K. Jiao, M. L. Zhang, S. M. Wang and C. Y. Yan, Int. J. Biol. Macromol., 2017, 101, 543-552.

5 T. G. Delgado-Leon, J. M. Salas-Pacheco, F. Vazquez-Alaniz, A. A. Vertiz-Hernandez, O. D. Lopez-Guzman, E. LozanoGuzman, A. Martinez-Romero, N. Urtiz-Estrada and M. Cervantes-Flores, J. Trace Elem. Med. Biol., 2018, 46, 144-149.

6 S. K. Park, Diabetes, 2017, 66, 1755-1757.

7 A. Ortega, G. Berna, A. Rojas, F. Martin and B. Soria, Int. J. Mol. Sci., 2017, 18, DOI: 10.3390/ijms18061188.

8 W. W. Feng, T. Zhao, G. H. Mao, W. Wang, Y. Feng, F. Li, D. H. Zheng, H. Y. Wu, D. Jin, L. Q. Yang and X. Y. Wu, PLoS One, 2015, 10, e0125952.

9 M. A. Brooks, J. L. Grimes, K. E. Lloyd, K. Krafka, A. Lamptey and J. W. Spears, Poult. Sci., 2016, 95, 1096-1104.

10 X. P. Yang, K. Palanichamy, A. C. Ontko, M. N. A. Rao, C. X. Fang, J. Ren and N. Sreejayan, FEBS Lett., 2005, 579, 1458-1464.

11 L. Liu, W. M. Cui, S. W. Zhang, F. H. Kong, M. A. Pedersen, Y. Wen and J. P. Lv, RSC Adv., 2015, 5, 3482-3490.

12 Y. Q. Wang and M. H. Yao, J. Nutr. Biochem., 2009, 20, 982991.

13 C. Hao, J. J. Hao, W. Wang, Z. R. Han, G. S. Li, L. J. Zhang, X. Zhao and G. L. Yu, PLoS One, 2011, 6, e24598.

14 W. Qiao, Z. L. Peng, Z. S. Wang, J. Wei and A. Zhou, Biol. Trace Elem. Res., 2009, 131, 133-142.

15 P. Zhao, J. Y. Wang, H. Ma, Y. Xiao, L. L. He, C. Tong, Z. H. Wang, Q. S. Zheng, E. K. Dolence, S. Nair, J. Ren and J. Li, Biochem. Pharmacol., 2009, 77, 1002-1010.

16 W. W. Feng, H. Y. Wu, Q. Li, Z. X. Zhou, Y. Chen, T. Zhao, Y. Feng, G. H. Mao, F. Li, L. Q. Yang and X. Y. Wu, Biol. Trace Elem. Res., 2015, 168, 181-195.

17 W. W. Feng, W. J. Zhang, T. Zhao, G. H. Mao, W. Wang, X. S. Wu, Z. X. Zhou, J. Huang, Y. T. Bao, L. Q. Yang and X. Y. Wu, Biol. Trace Elem. Res., 2015, 168, 150-168.

18 X. Y. Wu, F. Li, W. D. Xu, J. L. Zhao, T. Zhao, L. H. Liang and L. Q. Yang, Biol. Trace Elem. Res., 2011, 143, 1031-1043.

19 J. J. Guo, Y. Cao, C. T. Ho, S. K. Jin and Q. R. Huang, J. Funct. Foods, 2017, 34, 297-303.

20 P. J. Park, J. Y. Cho and E. G. Cho, Eur. J. Cell Biol., 2017, 96, 301-311.
21 G. H. Mao, Y. Ren, W. W. Feng, Q. Li, H. Y. Wu, D. Jin, T. Zhao, C. Q. Xu, L. Q. Yang and X. Y. Wu, Carbohydr. Polym., 2015, 134, 406-412.

22 X. Y. Xia, X. Xiang, F. H. Huang, M. M. Zheng, R. H. Cong, L. Han and Z. Zhang, RSC Adv., 2018, 43, 24338-24345.

23 A. F. Stattermayer, S. Traussnigg, E. Aigner, C. Kienbacher, U. Huber-Schonauer, P. Steindl-Munda, A. Stadlmayr, F. Wrba, M. Trauner, C. Datz and P. Ferenci, J. Trace Elem. Med. Biol., 2017, 39, 100-107.

24 M. Diaz, C. Garcia, G. Sebastiani, F. de Zegher, A. LopezBermejo and L. Ibanez, Diabetes, 2017, 66, 779-784.

25 Z. J. Fang, M. Zhao, H. Zhen, L. F. Chen, P. Shi and Z. W. Huang, PLoS One, 2014, 9, 1-9.

26 K. R. Manygoats, M. Yazzie and D. M. Stearns, J. Inorg. Chem., 2002, 7, 791-798.

27 L. Wang, Z. B. Liu, X. F. Liu and Y. T. Wu, RSC Adv., 2016, 93, 90777-90785.

28 M. Kim, K. Song and Y. S. Kim, Front. Pharmacol., 2017, 8, DOI: $10.3389 /$ fphar.2017.00405.

29 R. Costa, I. Rodrigues, L. Guardao, S. Rocha-Rodrigues, C. Silva, J. Magalhaes, M. Ferreira-de-Almeida, R. Negrao and R. Soares, J. Nutr. Biochem., 2017, 45, 39-47.

30 R. Dhanya, A. D. Arya, P. Nisha and P. Jayamurthy, Front. Pharmacol., 2017, 8, DOI: 10.3389/fphar.2017.00336.

31 S. Y. Lee, F. Y. Lai, L. S. Shi, Y. C. Chou, I. C. Yen and T. C. Chang, Phytomedicine, 2015, 22, 477-486.

32 L. Jovanovic, M. Pantelic, R. Prodanovic, I. Vujanac, M. Duric, S. Tepavcevic, S. Vranjes-Duric, G. Koricanac and D. Kirovski, Biol. Trace Elem. Res., 2017, 180, 223-232.

33 M. Peng and X. P. Yang, J. Inorg. Chem., 2015, 146, 97-103.

34 F. M. Fauzi, C. M. John, A. Karunanidhi, H. Y. Mussa, R. Ramasamy, A. Adam and A. Bender, J. Ethnopharmacol., 2017, 197, 61-72.

35 P. H. Fang, M. Yu, L. Zhang, D. Wan, M. Y. Shi, Y. Zhu, P. Bo and Z. W. Zhang, Mol. Cell. Endocrinol., 2017, 448, 77-86.

36 Y. Q. Wang and M. H. Yao, J. Nutr. Biochem., 2009, 20, 982991.

37 N. Yu, X. Fang, D. D. Zhao, Q. Q. Mu, J. C. Zuo, Y. Ma, Y. Zhang, F. F. Mo, D. W. Zhang and G. J. Jiang, PLoS One, 2017, 12, DOI: 10.1371/journal.pone.0168980.

38 E. K. Song, Y. R. Lee, Y. R. Kim, J. H. Yeom, C. H. Yoo, H. K. Kim, H. M. Park, H. S. Kang, J. S. Kim, U. H. Kim and M. K. Han, Cell Rep., 2012, 1607-1619.

39 Y. X. Liu, A. Song, S. S. Zang, C. Wang, G. Y. Song, X. L. Li, Y. J. Zhu, X. Yu, L. Li, Y. Wang and L. Y. Duan, J. Ethnopharmacol., 2015, 162, 244-252.

40 O. Tsave, M. P. Yavropoulou, M. Kafantari, C. Gabriela, J. G. Yovos and A. Salifoglou, J. Inorg. Biochem., 2016, 163, 323-331. 